\title{
Role of hsa_circ_0066966 in proliferation and migration of hepatitis B virus-related liver cancer cells
}

\author{
YINGHUA YUAN, XIAOJIN YANG and DESHENG XIE \\ Department of Infectious Diseases, Shanghai Fifth People's Hospital, \\ Fudan University, Shanghai 200240, P.R. China
}

Received September 3, 2020; Accepted September 14, 2021

DOI: $10.3892 /$ etm.2021.11010

\begin{abstract}
A large proportion of liver cancer cases is caused by hepatitis B virus (HBV) infection. In recent years, an increasing number of reports have indicated that circular RNAs (circRNAs) exert regulatory effects in cancer development, whereas the role of circRNAs in HBV-positive liver cancer requires further investigation. In the present study, abnormally expressed circRNAs were identified in HBV-positive liver cancer cells through microarray analysis. A total of 1,493 differentially expressed circRNAs [absolute fold-change $(F C) \geq 2]$ in HBV-positive liver cancer cells were detected, of which 171 were upregulated and 1,322 were downregulated. Subsequently, Gene Ontology enrichment analysis indicated that the genes of dysregulated circRNAs were mainly involved in regulating Sertoli cell differentiation and development, as well as telomeric DNA binding. Kyoto Encyclopedia of Genes and Genomes pathway analysis revealed that most of these genes were enriched in cancer-related signaling pathways, including the MAPK and Hippo signaling pathways. Next, the expression levels of the top-10 dysregulated circRNAs were verified in HBV-positive liver cancer cells through reverse transcription-quantitative PCR. Among them, hsa_circ_0066966 had the highest absolute $\log _{2} \mathrm{FC}$ value and was abnormally increased in $\mathrm{HBV}$-positive liver cancer cells. Functional experiments further verified that knockdown of hsa_circ_0066966 had a significant inhibitory effect on the proliferation and migration of $\mathrm{HBV}$-positive liver cancer cells. By contrast, overexpression of hsa_circ_0066966 in HBV-negative liver cancer cells resulted in the opposite effect. In conclusion, in the present study, comprehensive circRNA profiling in HBV-positive liver cancer cells indicated that hsa circ_0066966 may regulate the progression of HBV-positive liver cancer.
\end{abstract}

Correspondence to: Dr Xiaojin Yang, Department of Infectious Diseases, Shanghai Fifth People's Hospital, Fudan University, 128 Ruili Road, Shanghai 200240, P.R. China

E-mail: yangxiaojing@5thhospital.com

Key words: microarray analysis, hsa_circ_0066966, hepatitis B virus, liver cancer

\section{Introduction}

Liver cancer ranks sixth among the most common cancer types globally and 905,677 new cases were diagnosed in 2020 (1). It is also one of the three major causes of cancer-related death, seriously threatening the health and safety of individuals worldwide, particularly in China and East Asia (2,3). Research indicated that liver cancer is mainly caused by long-term alcohol consumption, obesity, exposure to poisons, hepatitis $\mathrm{C}$ virus and, importantly, hepatitis B virus (HBV) infection (4). HBV is a hepatotropic DNA virus with carcinogenic effects. Patients infected with HBV may develop serious diseases such as hepatitis, cirrhosis and even liver cancer. According to reports, at least 50-60\% of liver cancer cases worldwide are caused by persistent HBV infection (5). Furthermore, due to the high replication level and mutation rate of $\mathrm{HBV}$, the post-operative effects in HBV-positive patients with liver cancer are not ideal (6). Therefore, it is necessary to further analyze the occurrence and development mechanisms in HBV-positive liver cancer to identify novel therapeutic targets.

Circular RNAs (circRNAs) are newly discovered endogenous non-coding RNAs (ncRNAs) with a covalent closed-loop structure that are able to regulate gene transcription and are widely found in the cytoplasm of eukaryotic animals. As it contains neither 5' caps nor 3' polyadenylated tails, is not sensitive to RNase $\mathrm{R}$ digestion and is more stable than linear RNA (7). In recent years, a large body of experimental evidence has pointed out that the abnormal expression of circRNAs is closely related to various diseases, including cancer. CircRNAs have a dual regulatory role in cancer development, both as tumor suppressors (8) and as proto-oncogenes (9). In addition, certain circRNAs have been proven to actively participate in the pathogenesis of HBV-positive liver cancer, such as circ_0009582 (10) and circRNA_101764 (11). However, studies on circRNAs in HBV-positive liver cancer remain limited and further exploration is required.

In terms of biological functions, circRNAs regulate gene expression and protein translation by binding to RNA-binding proteins (12). For instance, in glioblastoma multiforme, circSMARCA5 interacts with SRSF1, negatively controls its expression and ultimately affects angiogenesis and VEGFA mRNA splicing (13). Furthermore, circRNAs may function as important competitive endogenous RNAs (ceRNAs) through microRNA (miRNA) response elements (MREs) to 
competitively adsorb miRNA and affect mRNA expression. For instance, the level of circRNA-UBE2G1 is significantly increased in osteoarthritis tissues and combines with miR-37 as a ceRNA to increase the expression of HIF-1 $\alpha$ and promote the development of osteoarthritis (14). CircHDAC9 participates in the occurrence and development of Alzheimer's disease by targeting the miR-138/Sirt1 pathway (15); circFAT1(e2) is able to act as a 'sponge' for miR-181b, inhibiting its expression, leading to HK2 imbalance and promoting osteosarcoma metastasis (16). However, the expression profile of dysregulated circRNAs in HBV-positive liver cancer and its underlying mechanisms remain to be fully elucidated.

In the present study, 1,493 differentially expressed circRNAs in HBV-positive liver cancer cells were screened through microarray analysis and the specific functions of differentially expressed circRNAs were analyzed through bioinformatics. Among the differentially expressed circRNAs, hsa_circ_0066966 was significantly increased in HBV-positive liver cancer cells. Finally, it was demonstrated that abnormal expression of hsa_circ_0066966 not only promoted the proliferation of HBV-positive liver cancer cells but also positively regulated their migration, which provided a novel perspective for the study of the mechanisms involved in HBV-positive liver cancer.

\section{Materials and methods}

Cell lines and culture. The four liver cancer cell lines used for the experimental part of the present study were all obtained from the Chinese Academy of Sciences, including two HBV-positive liver cancer cell lines (HepG2.2.15 and Hep3B) and two HBV-negative liver cancer cell lines (HepG2 and Huh7). All of the 4 cell lines had been authenticated by short tandem repeat profiling. According to the supplier's instructions, the cells were cultured in RPM1-1640 medium with $10 \%$ fetal bovine serum (FBS), $1 \%$ penicillin and $1 \%$ streptomycin (all from Invitrogen; Thermo Fisher Scientific, Inc.) at $37^{\circ} \mathrm{C}$ in an incubator with $5 \% \mathrm{CO}_{2}$.

Microarray analysis. The HBV-positive liver cancer cell lines HepG2.2.15 and Hep3B and the two HBV-negative liver cancer cell lines HepG2 and Huh7 were selected for the experiments performed in parallel. One experimental replicate was used for each cell line. In short, total RNA was isolated using the RNeasy Total RNA Isolation Kit (Qiagen $\mathrm{GmbH}$ ) according to the manufacturer's protocol and purified by using an RNeasy Mini Kit (Qiagen $\mathrm{GmbH}$ ). RNA samples were then used to generate biotinylated cRNA targets for the human ceRNA array v3.0 (Sinotech Genomics). The biotinylated cRNA targets were then hybridized with the slides. After hybridization, slides were scanned on the Agilent Microarray Scanner (Agilent Technologies, Inc.). Data were extracted with Feature Extraction software 10.7 (Agilent Technologies, Inc.). Raw data were normalized by a Quantile algorithm with the $\mathrm{R}$ package 'limma'. The circRNA data were then extracted from all of the normalized data. The dysregulated circRNAs in HBV-positive liver cancer cells and HBV-negative liver cancer cells were screened using a threshold of absolute fold-change $[\mathrm{FC}$ (abs)] $\geq 2$ and the resulting differentially expressed circRNAs were used for subsequent analysis. The original data of the microarray analysis were deposited as a Gene Expression Omnibus (GEO) dataset under the accession no. GSE181988 (https://www.ncbi.nlm.nih.gov/geo/query/acc. cgi?acc=GSE181988).

Annotation of circRNA/miRNA interactions. Arraystar's homemade miRNA target prediction software based on TargetScan (17) and miRanda (18) was employed to predict miRNAs that may interact with circRNAs. The output from the miRNA target prediction software also detailed annotations regarding circRNA/miRNA interactions.

Gene Ontology (GO) and Kyoto Encyclopedia of Genes and Genomes (KEGG) enrichment analyses. In order to further explore potential molecular mechanisms involving abnormal expression of circRNAs, GO and KEGG enrichment analyses of host genes of dysregulated circRNAs were performed using the $\mathrm{R}$ package clusterProfiler. $\mathrm{P}<0.05$ was considered to indicate statistically significant enrichment of these genes.

Reverse transcription-quantitative PCR (RT-qPCR). The levels of circRNAs in liver cancer cells were detected by RT-qPCR, with GAPDH used as an internal reference gene transcript. TRIzol reagent (Invitrogen; Thermo Fisher Scientific, Inc.) was used to extract total RNA from the four liver cancer cell lines as per the manufacturer's instructions, followed by RT of RNA into cDNA using the PrimeScript RT kit (Takara Bio, Inc.). Subsequently, according to the manufacturer's protocol, SYBR Premix Ex Taq ${ }^{\mathrm{TM}}$ (Takara Bio, Inc.) was used to perform qPCR on an Applied Biosystems 7500 Real-Time PCR platform (Applied Biosystems; Thermo Fisher Scientific, Inc.) using the following thermocycling conditions: Initial denaturation at $95^{\circ} \mathrm{C}$ for $5 \mathrm{~min}$, followed by 40 cycles of $95^{\circ} \mathrm{C}$ for $5 \mathrm{sec}$ and $60^{\circ} \mathrm{C}$ for $1 \mathrm{~min}$. Finally, the expression levels of circRNAs in liver cancer cells were identified using the $2^{-\Delta \Delta \mathrm{Cq}}$ method (19). The PCR primer sequences for the 10 circRNAs are listed in Table SI.

Cell transfection. Small interfering RNA (siRNA) against hsa_circ_0066966 (si-hsa_circ_0066966) and negative control siRNA (si-NC) were acquired from GenePharma. hsa_circ_0066966 expression plasmid (named as hsa_ circ_0066966) and its corresponding negative control (named NC) were obtained from Guangzhou RiboBio Co., Ltd. The liver cancer cells were first seeded in a 6-well plate and then cultured until 60-70\% confluent. Next, the plasmids and oligonucleotides were transfected into $\mathrm{HBV}$-negative liver cancer cells and HBV-positive liver cancer cells using Lipofectamine $200{ }^{\circledR}$ reagent (Invitrogen; Thermo Fisher Scientific, Inc.) following the manufacturer's protocols. The cells were harvested $48 \mathrm{~h}$ later and overexpression and interference efficiency were tested by RT-qPCR.

MTT assay. Cell proliferation ability after transfection was detected using the MTT assay (Invitrogen; Thermo Fisher Scientific, Inc.). In brief, transfected cells were seeded into a 96-well flat-bottom plate at a density of $4 \times 10^{3}$ cells/well and incubated for $24,48,72$ or $96 \mathrm{~h}$, followed by treatment with MTT at $37^{\circ} \mathrm{C}$ under $5 \% \mathrm{CO}_{2}$ for $4 \mathrm{~h}$. Finally, the medium in each well was removed and dimethyl sulfoxide was added. The 
Table I. Top 10 dysregulated circRNAs in hepatitis B virus-positive liver cancer cells.

\begin{tabular}{|c|c|c|c|c|c|}
\hline CircRNA & $\log _{2} \mathrm{FC}$ & $\mathrm{FC}$ & $\mathrm{FC}$ abs & $\begin{array}{l}\text { Direction of } \\
\text { dysregulation }\end{array}$ & Host gene \\
\hline hsa_circ_0030525 & -6.354 & 0.012 & 81.771 & Down & MYCBP2 \\
\hline hsa_circ_0079954 & 5.091 & 34.087 & 34.087 & Up & HECW1 \\
\hline hsa_circ_0090095 & -4.953 & 0.032 & 30.970 & Down & ACOT9 \\
\hline hsa_circ_0060534 & 4.306 & 19.787 & 19.787 & Up & SLPI \\
\hline hsa_circ_0032138 & -4.163 & 0.056 & 17.911 & Down & HIF1A \\
\hline hsa_circ_0066966 & 3.950 & 15.460 & 15.460 & Up & GOLGB1 \\
\hline hsa_circ_0085289 & -3.889 & 0.068 & 14.812 & Down & CTHRC1 \\
\hline hsa_circ_0088524 & 3.876 & 14.682 & 14.682 & $\mathrm{Up}$ & GOLGA1 \\
\hline hsa_circ_0062852 & -3.858 & 0.069 & 14.498 & Down & MORC2 \\
\hline hsa_circ_0091095 & -3.666 & 0.079 & 12.696 & Down & ATRX \\
\hline
\end{tabular}

FC, fold change; abs, absolute; circ, circular RNA.

optical absorbance at $492 \mathrm{~nm}$ was recorded using an ELX-800 University Microplate Reader (BioTek, Inc.).

Cell migration assay. Cell migration was detected using Transwell $^{\circledR}$ assays. After transfection, $5 \times 10^{4}$ cells were resuspended in $200 \mu \mathrm{l}$ RPMI 1640 medium without FBS and then cultured in the upper Transwell chambers $(8 \mu \mathrm{m}$ pore size; EMD Millipore). At the same time, RPMI 1640 medium with $10 \%$ FBS (as a chemoattractant) was placed into the lower Transwell chambers. After incubating the chamber at $37^{\circ} \mathrm{C}$ and $5 \% \mathrm{CO}_{2}$ for $24 \mathrm{~h}$, liver cancer cells in the upper chamber were removed and cells that had migrated to the bottom of the membrane were stained with $0.1 \%$ crystal violet $(20 \%$ methanol) for $15 \mathrm{~min}$ at room temperature. Finally, the cells were imaged using a microscope (Olympus Corporation) and five fields were randomly selected for cell counting.

Statistical analysis. SPSS v.21.0 (IBM Corporation) was used for data analysis. Values are expressed as the mean \pm standard deviation. Differences between the two groups were analyzed using Student's t-test. $\mathrm{P}<0.05$ was considered to indicate a statistically significant difference.

\section{Results}

Differentially expressed circRNAs between $H B V$-positive liver cancer cells and HBV-negative liver cancer cells. Heat-map analysis indicated that, compared with HBV-negative liver cancer cells, a total of 1,493 abnormally expressed circRNAs [FC (abs) $\geq 2$ ] were present in HBV-positive liver cancer cells, of which 171 were upregulated and 1,322 were significantly decreased (Fig. 1). In the present experiments, two types of liver cancer cell lines were used in the HBV-positive/negative liver cancer cell groups, hence it was not possible to obtain P-values involving changes in circRNA expression. Table I lists detailed information concerning the top-10 dysregulated circRNAs in HBV-positive liver cancer cells and their cognate genes. Among these, hsa_circ_0079954 exhibited the greatest level of upregulation [FC (abs)=34.087], while hsa_circ_0030525 was the most downregulated circRNA [FC (abs)=81.771]. Their host genes were HECW and MYCBP2, respectively. In addition, the cognate gene of hsa_circ_0066966, golgin B1 (GOLGB1), and the host gene of hsa_circ_0088524,GOLGA1, are both Golgi-related proteins.

Potential circRNA/miRNA interactions. To investigate the possible mechanisms involved in differentially expressed circRNAs in HBV-positive liver cancer cells, the MREs of the top-10 dysregulated circRNAs in HBV-positive liver cancer cells were predicted using the miRNA target prediction software from Arraystar Inc. based on TargetScan and miRanda and the top-10 dysregulated circRNAs were annotated in detail using the circRNA/miRNA interaction information (Table II). According to the results, the most upregulated circRNA, hsa_circ_0079954, targeted the MREs of the following miRNAs: hsa-miR-383-3p, hsa-miR-7161-3p, hsa-miR-3065-3p, hsa-miR-127-5p and hsa-miR-4291. Similarly, the most downregulated circRNA, hsa_circ_0030525, targeted the MREs of the following miRNAs: hsa-miR-391, hsa-miR-487a-5p, hsa-miR-27b-3p, hsa-miR-1273g-5p and hsa-miR-199a-5p. It is also worth noting that both hsa_circ_0066966 and hsa_circ_0088524 interacted with hsa-miR-3619-5p.

GO enrichment analysis of genes encoding dysregulated circRNAs. In order to annotate and classify circRNAs and to further understand the important biological roles of abnormally expressed circRNAs, GO enrichment analysis was performed on the cognate genes of differentially expressed circRNAs. Fig. 2 lists the top-30 enriched GO terms. GO analysis suggested that in the category molecular function, transmembrane receptor protein tyrosine phosphatase activity, telomeric DNA binding, proline-rich region binding, nucleocytoplasmic transporter activity and microtubule plus-end binding were the most enriched terms. In the category biological process, genes were mainly enriched in Sertoli cell differentiation and development, responses to leucine, regulation of sister chromatid cohesion and protein localization to chromosomes and 
A

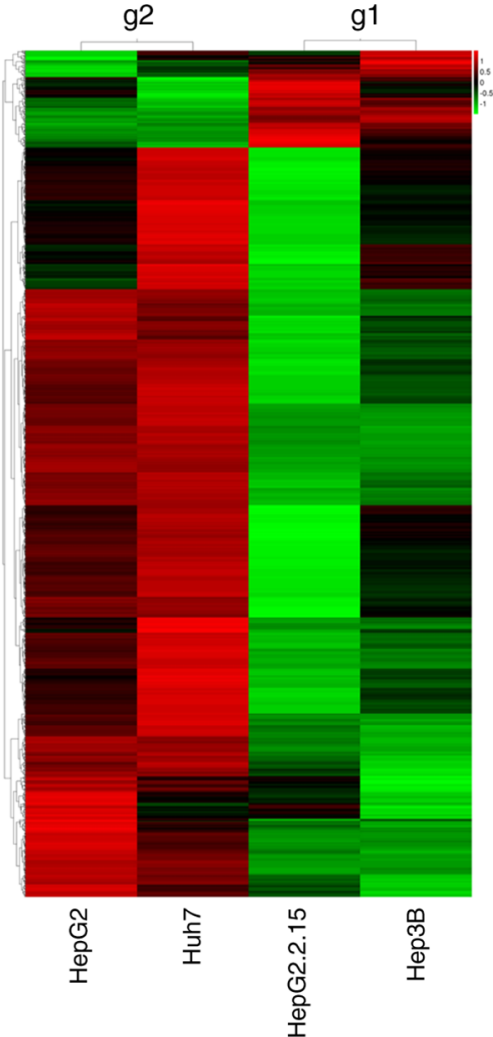

B

\author{
g1 VS g2
}

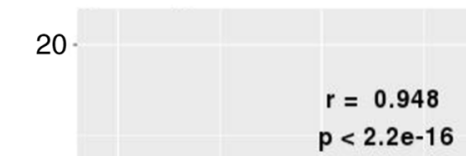

15

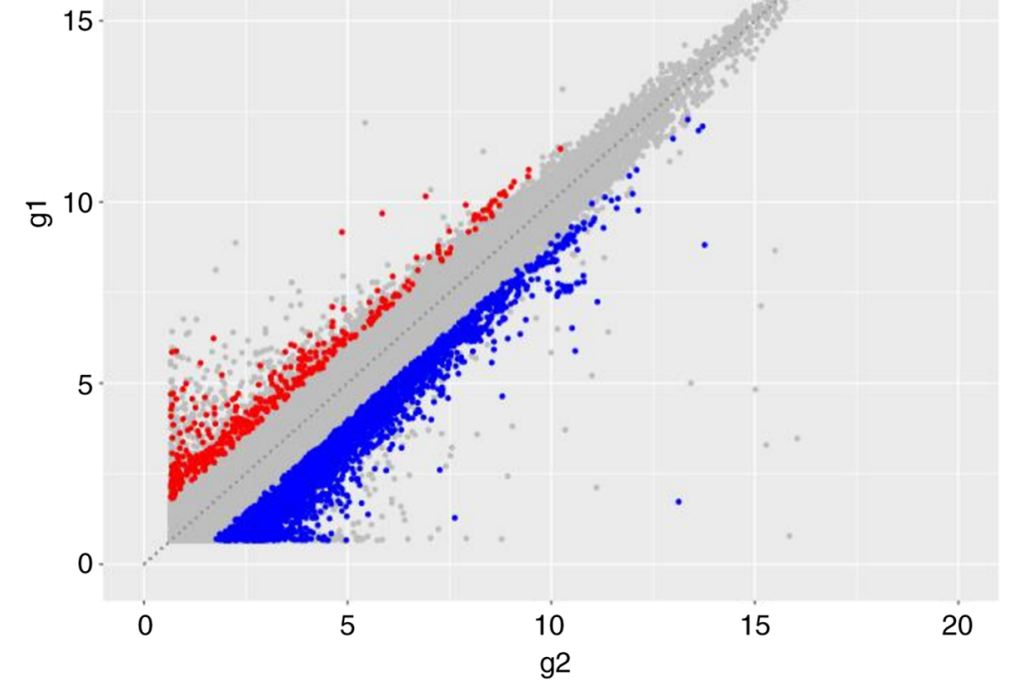

Figure 1. Identification of dysregulated circRNAs in HBV-positive live cancer cells. (A) Heat-map and (B) plot of dysregulated circRNAs in HBV-positive liver cancer cells analyzed by circRNA microarray (absolute fold-change $\geq 2$ ). The 1,493 differentially expressed circRNAs in HBV-positive and -negative liver cancer cells are presented. Red, upregulated; green, downregulated. g1, HBV-positive liver cancer cell group; g2, HBV-negative liver cancer cell group; circRNA, circular RNA; HBV, hepatitis B virus.

telomeric regions. In the category cellular component, genes were mainly enriched in the ISWI-type complex (Fig. 2).

KEGG pathway enrichment analysis of dysregulated circRNA cognate genes. KEGG pathway enrichment analysis was performed on the cognate genes of dysregulated circRNAs to further explore the signaling pathways that differentially expressed circRNAs may be involved in. Fig. 3 lists the top-30 pathways of the KEGG enrichment. The results indicated that pathways such as non-homologous end-joining, homologous recombination, Hippo signaling pathway in multiple species, cell cycle and MAPK signaling were significantly enriched. Among these, the cell cycle, MAPK and Hippo signaling pathways are related to a variety of cancers. In addition, pathways directly related to bladder cancer were also enriched (Fig. 3), which implies that the differentially expressed circRNAs identified have a high functional correlation with cancer.

$R T$-qPCR validation of the top-10 dysregulated circRNAs in $H B V$-positive liver cancer cells. To further support the reliability of the microarray results, the expression of the top-10 dysregulated circRNAs in HBV-positive liver cancer cells was verified by RT-qPCR. The results indicated that only the expression trend of hsa_circ_0090095 was opposite to the microarray results. The expression trends of the remaining 9 circRNAs (hsa_circ_0030525, hsa_circ_0079954, hsa_circ_0060534, hsa_circ_0032138hsa_circ_0066966hsa_circ_0085289hsa_circ_0088524, hsa_circ_0062852andhsa_circ_0091095) were similar to their microarray results, and the values of $\log _{2} \mathrm{FC}$ of the circRNAs were not significantly different between the RT-qPCR and microarray data $(P>0.05)$, indicating that the microarray analysis results were reliable. In addition, the absolute value of $\log _{2}$ FC of hsa_circ_0066966 was the highest (Fig. 4), and thus, this circRNA was used for further research.

Knockdown of hsa_circ_0066966 in HBV-positive liver cancer cells inhibits cellular proliferation and migration. hsa_circ_0066966 (chr3:121441108-121448187) was derived from the GOLGB1 gene. The function of hsa_circ_0066966 in HBV-positive liver cancer cells was explored. Si-hsa circ_0066966 was transfected into the corresponding liver cancer cells and RT-qPCR was used to verify the knockdown efficiency. The results indicated that si-hsa_circ_0066966 transfection significantly reduced hsa_circ_0066966 levels in HepG2.2.15 and Hep3B cells ( $\mathrm{P}<0.05$; Fig. 5A). Next, MTT assays were performed and it was revealed that in the two HBV-positive liver cancer cell lines, hsa_circ_0066966 silencing significantly inhibited cell proliferation at $96 \mathrm{~h}$ $(\mathrm{P}<0.05$; Fig. 5B). The experiment was followed by Transwell assays, which indicated that, compared with the si-NC group, hsa_circ_0066966 silencing significantly inhibited the migration ability of HepG2.2.15 and Hep3B cells $(\mathrm{P}<0.05$; Fig. 5C). The above results demonstrated that knockdown of hsa_circ_0066966 not only inhibited the proliferation of HBV-positive liver cancer cells but also affected their migration. 
Table II. MREs of top 10 dysregulated circRNAs in hepatitis B virus-positive liver cancer cells.

\begin{tabular}{llllll}
\hline CircRNA & \multicolumn{1}{c}{ MRE1 } & \multicolumn{1}{c}{ MRE2 } & \multicolumn{1}{c}{ MRE3 } & \multicolumn{1}{c}{ MRE4 } & MRE5 \\
\hline hsa_circ_0030525 & hsa-miR-3918 & hsa-miR-487a-5p & hsa-miR-27b-3p & hsa-miR-1273g-5p & hsa-miR-199a-5p \\
hsa_circ_0079954 & hsa-miR-383-3p & hsa-miR-7161-3p & hsa-miR-3065-3p & hsa-miR-127-5p & hsa-miR-4291 \\
hsa_circ_0090095 & hsa-miR-7153-5p & hsa-miR-8070 & hsa-miR-3925-5p & hsa-miR-455-3p.1 & hsa-miR-212-3p \\
hsa_circ_0060534 & hsa-miR-4480 & hsa-miR-6791-3p & hsa-miR-4776-5p & hsa-miR-6165 & hsa-miR-664a-3p \\
hsa_circ_0032138 & hsa-miR-5708 & hsa-miR-22-5p & hsa-miR-6504-5p & hsa-miR-664a-5p & hsa-miR-149-5p \\
hsa_circ_0066966 & hsa-miR-214-3p & hsa-miR-922 & hsa-miR-646 & hsa-miR-3619-5p & hsa-miR-374a-3p \\
hsa_circ_0085289 & hsa-miR-338-3p & hsa-miR-29a-3p & hsa-miR-5586-5p & hsa-miR-6507-3p & hsa-miR-29c-3p \\
hsa_circ_0088524 & hsa-miR-3689e & hsa-miR-6740-3p & hsa-miR-6809-3p & hsa-miR-3619-5p & hsa-miR-3689b-5p \\
hsa_circ_0062852 & hsa-miR-4277 & hsa-miR-514b-3p & hsa-miR-584-3p & hsa-miR-514a-3p & hsa-miR-409-3p \\
hsa_circ_0091095 & hsa-miR-377-3p & hsa-miR-3133 & hsa-miR-4511 & hsa-miR-5195-3p & hsa-miR-145-5p
\end{tabular}

MRE, miRNA response element; miR, microRNA; circ, circular RNA.

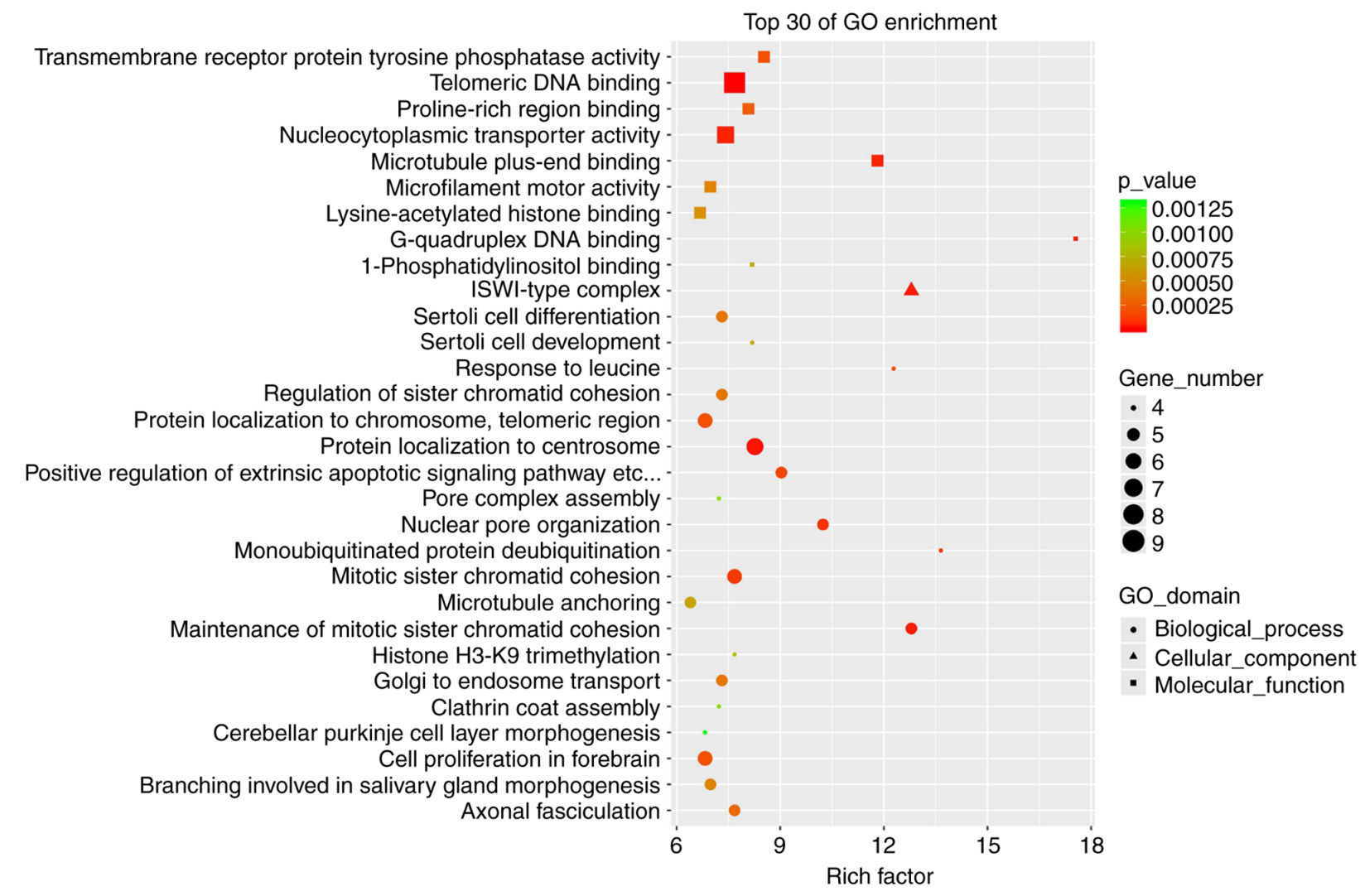

Figure 2. GO enrichment analysis of genes encoding dysregulated circRNAs. The top-30 GO enrichments are listed. The sizes of circles, triangles, and squares indicate the number of differentially expressed circRNAs involved in biological processes, cell components, and molecular functions. The greater the size, the more genes. The color represents P-values; the redder the color, the smaller the P-value, and the more significant the enrichment. GO, gene ontology; circRNA, circular RNA.

Overexpression of hsa_circ_0066966 in HBV-negative liver cancer cells promotes cellular proliferation and migration. Finally, in order to explore whether hsa_circ_0066966 has a role in HBV-negative liver cancer cells, an hsa_circ_0066966 overexpression plasmid was constructed. The results confirmed that after cells were transfected with hsa_circ_0066966 plasmid, the levels of hsa_circ_0066966 increased significantly $(\mathrm{P}<0.05$; Fig. 6A). According to the results of the MTT assays, hsa_circ_0066966 overexpression in HepG2 and Huh7 cells significantly promoted the proliferative capacity at $96 \mathrm{~h}(\mathrm{P}<0.05$; Fig. 6B). It was then explored whether hsa_circ_0066966 is able to regulate the migration of HBV-negative liver cancer cells. The Transwell assays indicated that, compared with the NC group, the migration ability of HBV-negative liver cancer cells in the hsa_circ_0066966 group was significantly enhanced $(\mathrm{P}<0.05$; Fig. 6C). In conclusion, overexpression of hsa_circ_0066966 had a significant stimulatory effect on the proliferation and migration of HBV-negative liver cancer cells. 


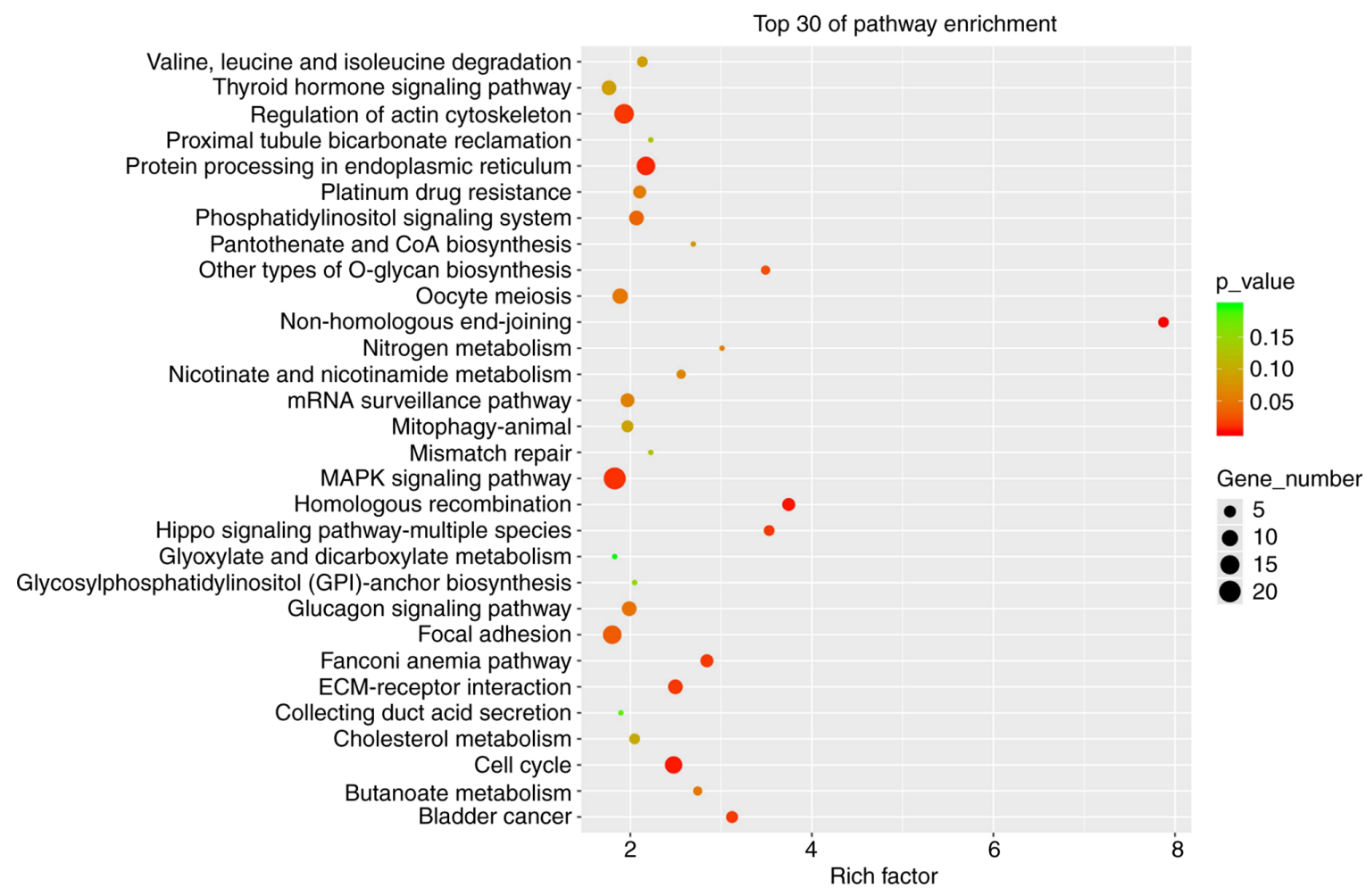

Figure 3. KEGG pathway enrichment analysis of cognate genes of dysregulated circular RNAs. The top-30 KEGG pathways are listed. The value of the rich factor is proportional to the degree of enrichment. The P-values are represented by color (the redder the color, the smaller the P-value); the size of the circles represents the number of differential genes under the term. KEGG, Kyoto Encyclopedia of Genes and Genomes.

\section{Discussion}

CircRNAs are a type of stable ncRNA. Continuous research has pointed out that circRNAs have vital roles in a considerable number of diseases, particularly cancer. For instance, hsa_circRNA_000166 has a significant promoting effect on migration and invasion by colon cancer cells (20) and hsa_circ_0131242 positively regulates the occurrence of triple-negative breast cancer through sponging hsa-miR-2682 (21). In addition, other studies further demonstrated that circCYFIP2 affects the level of E2F1 by regulating miR-1205 and ultimately mediates metastasis in gastric cancer (22). Due to the gradual increase in morbidity and mortality of HBV-positive liver cancer cases, there is an urgent requirement to identify key targets in the pathogenesis of HBV-positive liver cancer. Therefore, exploring the expression of circRNAs in HBV-positive liver cancer may further augment therapeutic approaches. In the present experiments, by determining the expression of dysregulated circRNAs in HBV-positive liver cancer cells through microarray analysis, a total of 1,493 differentially expressed circRNAs [FC (abs) $\geq 2$ ] were identified, of which 171 were significantly increased; the remaining 1,322 were abnormally decreased, indicating that abnormal expression of circRNAs was significantly associated with the occurrence of HBV-positive liver cancer.

In the microarray analysis, only two samples were included in each group, unlike the usually used 3 samples or 3 repeats. In fact, it was intended to compare 3 types of HBV-positive liver cancer cells with $3 \mathrm{HBV}$-negative liver cancer cells, but the third HBV-positive liver cancer cell line was not possible to obtain. In addition, compared to using three replicate

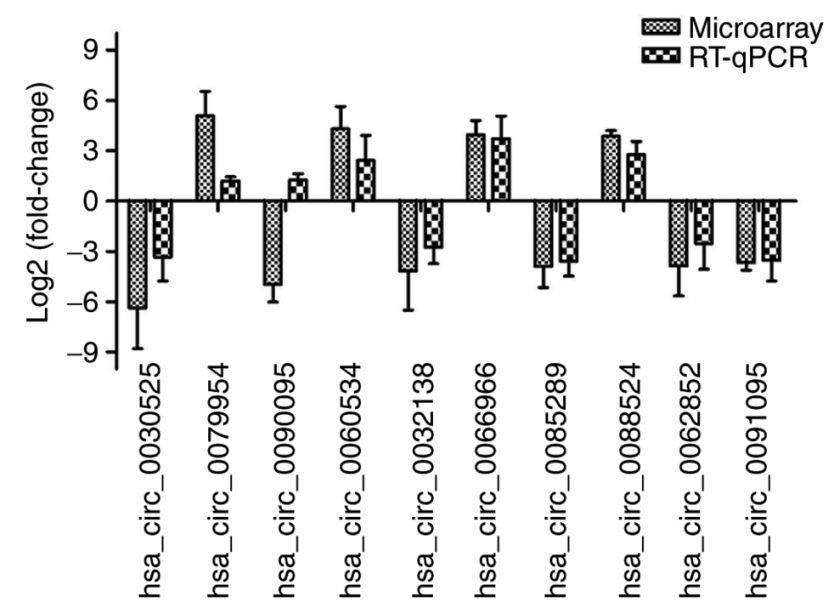

Figure 4. RT-qPCR analysis of the expression of the top-10 dysregulated circRNAs in HBV-positive liver cancer cells $(n=3)$. RT-qPCR, reverse transcription-quantitative PCR; circ, circular RNA.

samples, using two different samples in each group improves the screening of those circRNAs exerting important oncogenic roles. Therefore, two different cell lines were used for microarray in each group.

As is widely known, circRNAs with MREs are able to bind and adsorb the corresponding miRNA molecules through competitive binding, thereby exerting a 'sponging' effect to reduce the negative regulation of miRNA on gene expression and thus regulating the expression of target genes (12). For instance, circRNA 000554 has an inhibitory effect on epithelial-mesenchymal transition in breast cancer and regulates 
A

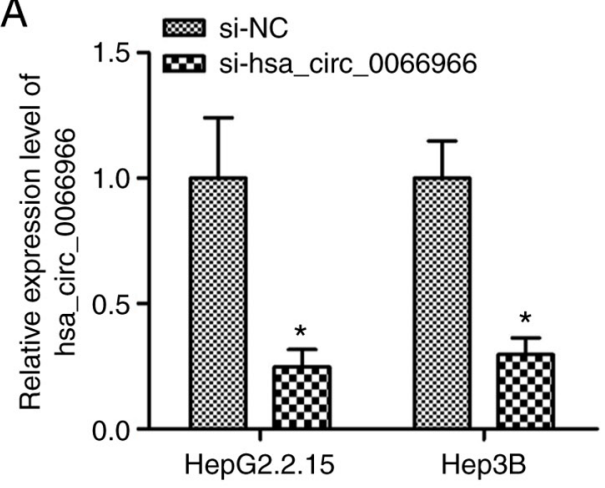

C

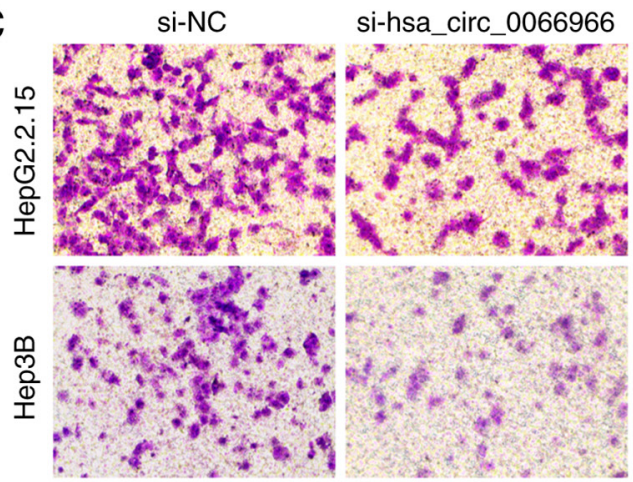

$\mathrm{B}$
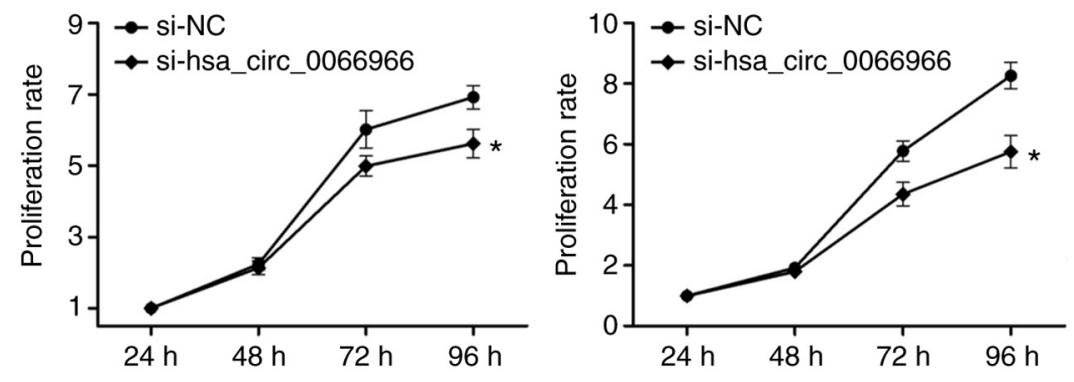

Figure 5. Knockdown of hsa_circ_0066966 in HBV-positive liver cancer cells inhibits cellular proliferation and migration. (A) After knockdown of hsa_circ_0066966, the levels of hsa_circ_0066966 in HepG2.2.15 and Hep3B cells were analyzed by reverse transcription-quantitative PCR. (B) The effects of downregulation of hsa_circ_0066966 on the proliferation of HBV-positive liver cancer cells were investigated using MTT assays. (C) The effects of downregulation of hsa_circ_0066966 on the migration of HBV-positive liver cancer cells were measured using Transwell migration assays (magnification, x200). ${ }^{P} \mathrm{P}<0.05$ vs. si-NC ( $\mathrm{n}=3$ in each experiment). circ, circular RNA; si-hsa_circ_0066966, small inhibitory RNA targeting hsa_circ_0066966; NC, negative control; HBV, hepatitis B virus.

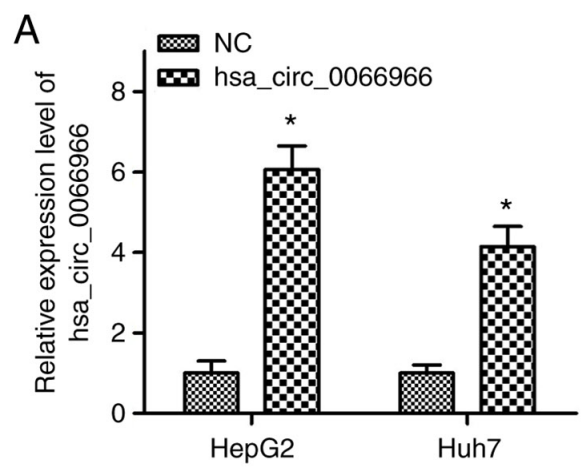

B

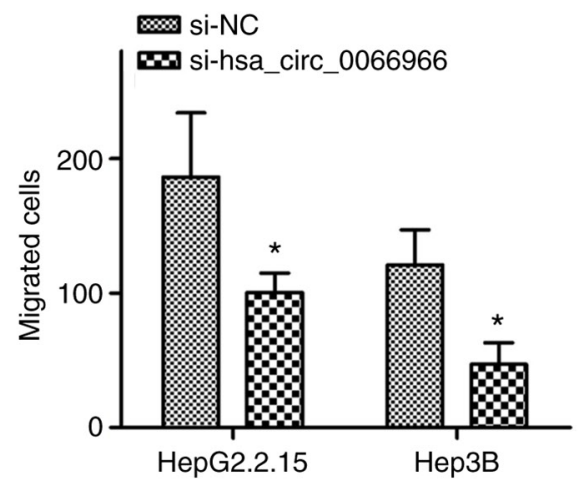

C
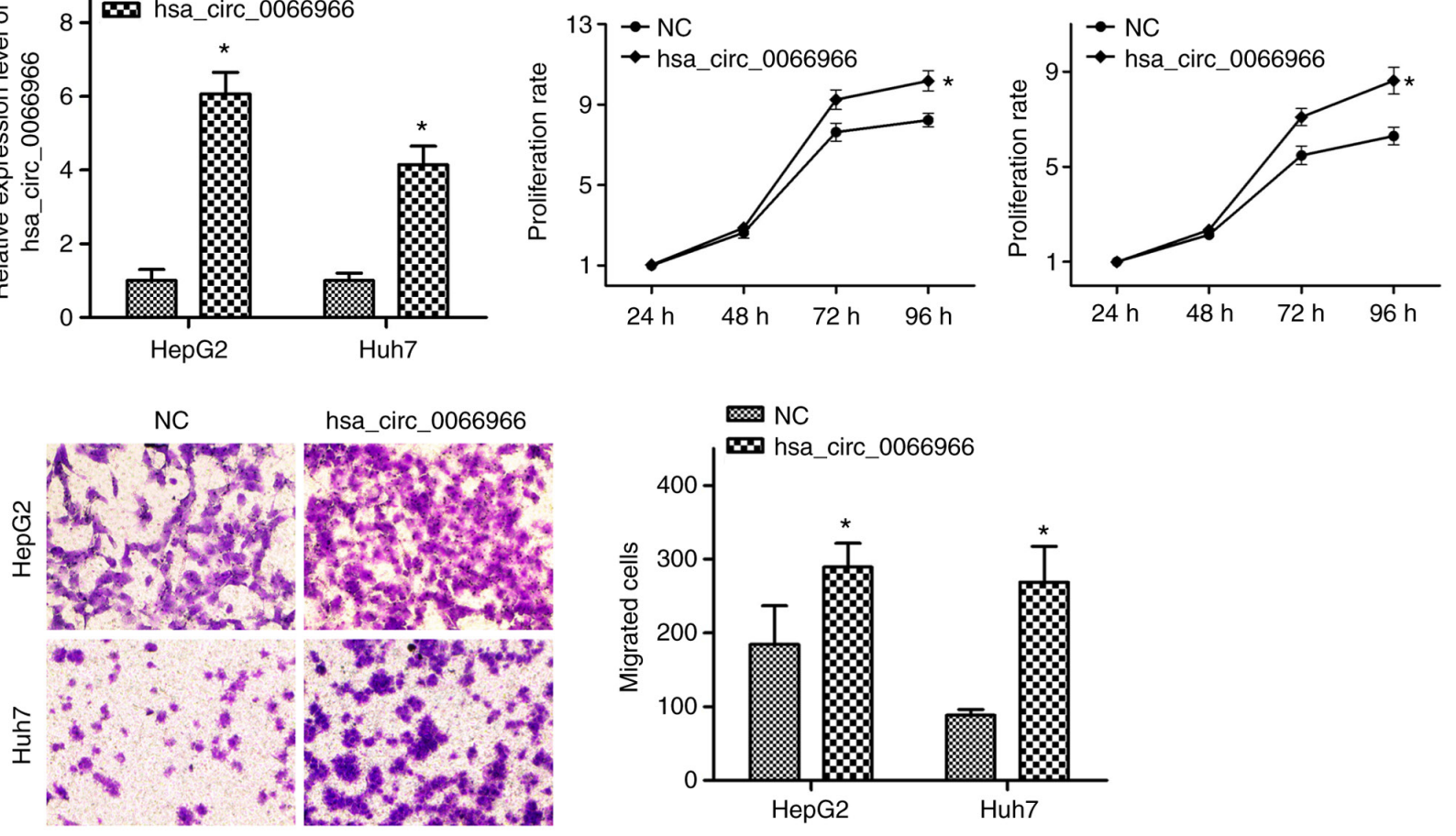

Figure 6. Overexpression of hsa_circ_0066966 in HBV-negative liver cancer cells promotes cellular proliferation and migration. (A) After hsa_circ_0066966 overexpression plasmid transfection into HepG2 and Huh7 cells, hsa_circ_0066966 levels in HepG2 and Huh7 cells were analyzed by reverse transcription-quantitative PCR. (B) The effects of overexpression of hsa_circ_0066966 on the proliferation of HBV-negative liver cancer cells were evaluated using MTT assays. (C) The effects of hsa_circ_0066966 overexpression on the migration of HBV-negative liver cancer cells were measured by Transwell assays (magnification, x200). ${ }^{~} \mathrm{P}<0.05$ vs. NC ( $\mathrm{n}=3$ in each experiment). circ, circular RNA; NC, negative control; HBV, hepatitis B virus. 
the level of ZFP36 via its sponging effect on miR-182 (23). Furthermore, circPACRGL, derived from exosomes, regulates the expression of TGF- $\beta 1$ through competitive binding with miR-142-3p and miR-506-3p, and promotes colorectal cancer progression (24). In the present study, the five MREs of the top-10 differentially expressed circRNAs were predicted by bioinformatics analysis. Of note, hsa_circ_0066966 and hsa_circ_0088524 shared the same MRE, hsa-miR-3619-5p, which has been proven to have tumor-suppressive effects in cisplatin-resistant cutaneous squamous cell carcinoma and bladder cancer $(25,26)$. Furthermore, Tan et al $(27)$ predicted that circZFR may be used as a 'sponge' for hsa-miR-3619-5p and promote the development of liver cancer. Hence, it was speculated that hsa_circ_0066966 and hsa_circ_0088524 simultaneously competitively bind hsa-miR-3619-5p to inhibit its expression and accelerate the processes of HBV-positive liver cancer. In addition, two other MREs, hsa-miR-214-3p and hsa-miR-646, of hsa_circ_0066966 were also reported to be closely involved in liver cancer progression. For instance, hsa-miR-214-3p has been indicated to be sponged by lncRNA HOXA11-AS (28) and hsa_circ_0008450 (29), which has an oncogenic role in liver cancer tumorigenesis, and ectopic expression of hsa-miR-214-3p suppressed liver cancer cell proliferation and invasion (28). Another study indicated that hsa-miR-646 is an MRE of circ_0000267 and co-transfection with circ_0000267 and miR-646 significantly reversed the oncogenic biological behavior of liver cancer cells induced by circ_0000267 (30). Therefore, the two MREs may be a potential means for hsa_circ_0066966 to exert its oncogenic role in HBV-positive liver cancer.

To further clarify the role of differentially expressed circRNAs in HBV-positive liver cancer, GO and KEGG pathway enrichment analysis was performed. GO enrichment analysis revealed that the most significantly enriched items were Sertoli cell differentiation and development and telomeric DNA binding, indicating that the differentially expressed circRNAs may participate in these biological functions. In addition, KEGG pathway enrichment analysis revealed that pathways related to cancer were significantly enriched, such as the MAPK and Hippo signaling pathways. The triggering of liver cancer involves activation of the MAPK signaling pathway (31). Furthermore, the Hippo signaling pathway was reported to have a certain relationship with the occurrence and development of liver cancer (32). Another study indicated that the circular RNA LPAR3 affected the migration of esophageal squamous cell carcinoma cells by activating the MAPK signaling pathway, while regulating cellular invasion (33). At the same time, hsa_circ_0128846 regulates the MST1, LATS1 and YAP proteins in the Hippo signaling pathway, ultimately promoting the progression of colorectal cancer (34). In the present study, the MAPK and Hippo signaling pathways significantly enriched cognate circRNA genes with dysregulated expression, indicating that circRNA may have a role in the progression and prognosis of HBV-positive liver cancer by regulating the MAPK and Hippo pathways.

Next, the expression of the top-10 dysregulated circRNAs in HBV-positive liver cancer cells was verified. The RT-qPCR results indicated that among the top-10 dysregulated circRNAs identified by microarray analysis, only the expression trend of hsa_circ_0090095 was opposite to the microarray results, suggesting that the microarray results were reliable. It also determined that hsa_circ_0066966 had the highest absolute value of $\log _{2} \mathrm{FC}$, with significantly increased levels in HBV-positive liver cancer cells. Although there is no relevant literature regarding the function of hsa_circ_0066966, GOLGB1, the gene encoding hsa circ_0066966, has been proven to positively influence the occurrence and development of liver cancer (35). Therefore, hsa_circ_0066966 was speculated to regulate the progression of liver cancer. CircRNAs have been demonstrated to regulate the proliferation and migration of cancer cells to varying degrees. For instance, hsa-circRNA-103809 promoted the proliferation of liver cancer cells by miR-1270 (36) and circ_0091579 may also actively regulate proliferation, migration and invasion of liver cancer cells (37). In the present study, the function of hsa_circ_0066966 in HBV-positive and -negative liver cancer cells was investigated. The results suggested that if the expression of hsa_circ_0066966 was diminished, a decrease in the proliferation and migration ability of HBV-positive liver cancer cells was observed. By contrast, increased expression of hsa_circ_0066966 not only enhanced the proliferative capacity of $\mathrm{HBV}$-negative liver cancer cells but also significantly improved their migratory ability. These results are consistent with those of a previous study, suggesting that hsa_circ_0066966's cognate gene positively regulates the proliferation and migration of HBV-positive liver cancer cells and has an inhibitory effect on apoptosis (35), which may be a potential mechanism involved in HBV-positive liver cancer.

To date, it remains elusive how HBV influences circRNA expression, but the important roles of the major protein of $\mathrm{HBV}, \mathrm{HBx}$, in cellular transcriptional regulation may provide certain clues to this. First, a previous genome-wide analysis of HBx chromatin recruitment in HBV-replicating cells (38) revealed that there are specific loci for HBx to bind to a large number of target sequences, including protein-coding genes and ncRNAs, which are enriched in pathways of cell metabolism, chromatin dynamics and cancer (38). Furthermore, $\mathrm{HBx}$ is able to interact with multiple transcription factors, including ATF/CREB, ATF3, c/EBP, NF-IL-6, ETS, EGR, SMAD4, OCT1, RXR receptor, p53, chromatin-modifying enzymes (CBP, p300 and PCAF) and components of the basal transcriptional machinery (RPB5, TFIIB, TBP and TFIIH) (39). The extensive functions of HBx indicate that alterations to the expression of circRNAs in HBV-positive cells may be attributed to the direct regulation of $\mathrm{HBx}$ on certain circRNAs or interactions with transcription factors, directly regulating the transcription of circRNAs, which awaits further investigation.

However, it should be acknowledged that the present study had certain limitations. First, the sample number used for microarray was $<3$ in each group and one additional HBV-negative and HBV-positive liver cancer cell line may be used for future studies. In addition, the specific mechanisms involving hsa circ_0066966 still require to be investigated. In the meantime, it may be useful to perform related animal experiments and further demonstrate the clinical significance.

In summary, the present study identified dysregulated circRNAs in HBV-positive liver cancer cells through microarray analysis and screened 171 circRNAs with significantly 
increased expression and 1,322 circRNAs with significantly decreased expression. In addition, it was demonstrated that hsa_circ_0066966 was abnormally expressed in HBV-positive liver cancer cells and was closely related to liver cancer cell proliferation and migration. Therefore, hsa_circ_0066966 may provide novel research directions for investigating mechanisms involving HBV-positive liver cancer in the future.

\section{Acknowledgements}

Not applicable.

\section{Funding}

This work was supported by the Natural Science Research Project of the Shanghai Minhang Science and Technology Committee (grant nos. 2018MHZ074 and 2019MHZ069).

\section{Availability of data and materials}

The datasets used and/or analyzed during the current study are available from the corresponding author on reasonable request. The original data of the microarray analysis were deposited as a GEO dataset under accession no. GSE181988 (https://www. ncbi.nlm.nih.gov/geo/query/acc.cgi?acc=GSE181988).

\section{Authors' contributions}

XY designed the study. YY performed the experiments and wrote the manuscript. XY revised the manuscript. YY and DX contributed to analyzing the data. XY and DX confirm the authenticity of all the raw data. All authors reviewed the results. All authors have read and approved the final manuscript.

\section{Ethics approval and consent to participate}

Not applicable.

\section{Patient consent for publication}

Not applicable.

\section{Competing interests}

The authors declare that they have no competing interests.

\section{References}

1. de Mattos AZ, Debes JD, Boonstra A, Yang JD, Balderramo DC, Sartori GDP and de Mattos AA: Current impact of viral hepatitis on liver cancer development: The challenge remains. World J Gastroenterol 27: 3556-3567, 2021.

2. Bravi F, Bosetti C, Tavani A, Gallus S and La Vecchia C: Coffee reduces risk for hepatocellular carcinoma: An updated meta-analysis. Clin Gastroenterol Hepatol 11: 1413-1421.e1, 2013.

3. Jemal A, Bray F, Center MM, Ferlay J, Ward E and Forman D: Global cancer statistics. CA Cancer J Clin 61: 69-90, 2011.

4. El-Serag HB and Rudolph KL: Hepatocellular carcinoma: Epidemiology and molecular carcinogenesis. Gastroenterology 132: 2557-2576, 2007.
5. Tangkijvanich $\mathrm{P}$, Mahachai V, Komolmit P, Fongsarun J, Theamboonlers A and Poovorawan Y: Hepatitis B virus genotypes and hepatocellular carcinoma in Thailand. World J Gastroenterol 11: 2238-2243, 2005.

6. Du Y, Su T, Ding Y and Cao G: Effects of antiviral therapy on the recurrence of hepatocellular carcinoma after curative resection or liver transplantation. Hepat Mon 12: e6031, 2012.

7. Meng S, Zhou H, Feng Z, Xu Z, Tang Y, Li P and Wu M: CircRNA: Functions and properties of a novel potential biomarker for cancer. Mol Cancer 16: 94, 2017.

8. He YX, Ju H, Li N, Jiang YF, Zhao WJ, Song TT and Ren WH: Association between hsa_circ_0006156 expression and incidence of gastric cancer. Eur Rev Med Pharmacol Sci 24: 3030-3036, 2020.

9. Chen KH, Pan JF, Chen ZX, Pan D, Gao T, Huang M and Huang JN: Effects of hsa_circ_0000711 expression level on proliferation and apoptosis of hepatoma cells. Eur Rev Med Pharmacol Sci 24: 4161-4171, 2020.

10. Wu C, Deng L, Zhuo H, Chen X, Tan Z, Han S, Tang J, Qian X and Yao A: Circulating circRNA predicting the occurrence of hepatocellular carcinoma in patients with HBV infection. J Cell Mol Med 24: 10216-10222, 2020.

11. Wang S, Cui S, Zhao W, Qian Z, Liu H, Chen Y, Lv F and Ding HG: Screening and bioinformatics analysis of circular RNA expression profiles in hepatitis B-related hepatocellular carcinoma. Cancer Biomark 22: 631-640, 2018.

12. Cortes-Lopez M and Miura P: Emerging functions of circular RNAs. Yale J Biol Med 89: 527-537, 2016.

13. Barbagallo D, Caponnetto A, Brex D, Mirabella F, Barbagallo C, Lauretta G, Morrone A, Certo F, Broggi G, Caltabiano R, et al: CircSMARCA5 regulates VEGFA mRNA splicing and angiogenesis in glioblastoma multiforme through the binding of SRSF1. Cancers (Basel) 11: 194, 2019.

14. Chen G, Liu T, Yu B, Wang B and Peng Q: CircRNA-UBE2G1 regulates LPS-induced osteoarthritis through miR-373/HIF-1a axis. Cell Cycle 19: 1696-1705, 2020.

15. Lu Y, Tan L and Wang X: Circular HDAC9/microRNA-138/ Sirtuin-1 pathway mediates synaptic and amyloid precursor protein processing deficits in Alzheimer's disease. Neurosci Bull 35: 877-888, 2019.

16. Gu H, Cheng X, Xu J, Zhou K, Bian C, Chen G and Yin X: Circular RNA circFAT1(e2) promotes osteosarcoma progression and metastasis by sponging miR-181b and Regulating HK2 Expression. Biomed Res Int 2020: 3589871, 2020.

17. Pasquinelli AE: MicroRNAs and their targets: Recognition, regulation and an emerging reciprocal relationship. Nat Rev Genet 13: 271-282, 2012.

18. Enright AJ, John B, Gaul U, Tuschl T, Sander C and Marks DS: MicroRNA targets in Drosophila. Genome Biol 5: R1, 2003.

19. Livak KJ and Schmittgen TD: Analysis of relative gene expression data using real-time quantitative PCR and the 2(-Delta Delta C(T)) method. Methods 25: 402-408, 2001.

20. Zhao G and Dai GJ: Hsa_circRNA_000166 Promotes cell proliferation, migration and invasion by regulating miR-330-5p/ELK1 in colon cancer. Onco Targets Ther 13: 5529-5539, 2020.

21. Li Y, Shi P, Zheng T, Ying Z and Jiang D: Circular RNA hsa circ_0131242 promotes triple-negative breast cancer progression by sponging hsa-miR-2682. Onco Targets Ther 13: 4791-4798, 2020.

22. Lin J, Liao S, Li E, Liu Z, Zheng R, Wu X and Zeng W: circCYFIP2 acts as a sponge of miR-1205 and affects the expression of its target gene E2F1 to regulate gastric cancer metastasis. Mol Ther Nucleic Acids 21: 121-132, 2020.

23. Mao Y, Lv M, Cao W, Liu X, Cui J, Wang Y, Wang Y, Nie G, Liu X and Wang H: Circular RNA 000554 represses epithelial-mesenchymal transition in breast cancer by regulating microRNA-182/ZFP36 axis. FASEB J: 34: 11405-11420, 2020.

24. Shang A, Gu C, Wang W, Wang X, Sun J, Zeng B, Chen C, Chang W, Ping Y, Ji P, et al: Exosomal circPACRGL promotes progression of colorectal cancer via the miR-142-3p/miR-506-3pTGF- $\beta 1$ axis. Mol Cancer 19: 117, 2020.

25. Zhang M, Luo H and Hui L: MiR-3619-5p hampers proliferation and cisplatin resistance in cutaneous squamous-cell carcinoma via KPNA4. Biochem Biophys Res Commun 513: 419-425, 2019.

26. Zhang Q, Miao S, Han X, Li C, Zhang M, Cui K, Xiong T, Chen Z, Wang C and Xu H: MicroRNA-3619-5p suppresses bladder carcinoma progression by directly targeting beta-catenin and CDK2 and activating p21. Cell Death Dis 9: 960,2018. 
27. Tan A, Li Q and Chen L: CircZFR promotes hepatocellular carcinoma progression through regulating miR-3619-5p/CTNNB1 axis and activating Wnt/ $\beta$-catenin pathway. Arch Biochem Biophys 661: 196-202, 2019.

28. Zhan M, He K, Xiao J, Liu F, Wang H, Xia Z, Duan X, Huang R, Li Y, He X, et al: LncRNA HOXA11-AS promotes hepatocellular carcinoma progression by repressing miR-214-3p. J Cell Mol Med 22: 3758-3767, 2018

29. Lin T, Dai Y, Guo X, Chen W, Zhao J, Cao L and Wu Z: Silencing of hsa_circ_0008450 represses hepatocellular carcinoma progression through regulation of microRNA-214-3p/EZH2 Axis. Cancer Manag Res 11: 9133-9143, 2019.

30. Pan H, Tang L, Jiang H, Li X, Wang R, Gao J and Li Q: Enhanced expression of circ_0000267 in hepatocellular carcinoma indicates poor prognosis and facilitates cell progression by sponging miR-646. J Cell Biochem: Feb 5, 2019 (Epub ahead of print).

31. Huang JL, Ren TY, Cao SW, Zheng SH, Hu XM, Hu YW, Lin L, Chen J, Zheng L and Wang Q: HBx-related long non-coding RNA DBH-AS1 promotes cell proliferation and survival by activating MAPK signaling in hepatocellular carcinoma. Oncotarget 6: 33791-33804, 2015.

32. Zhao X, Qin W, Jiang Y, Yang Z, Yuan B, Dai R, Shen H, Chen Y, $\mathrm{Fu} \mathrm{J}$ and Wang $\mathrm{H}$ : ACADL plays a tumor-suppressor role by targeting Hippo/YAP signaling in hepatocellular carcinoma. NPJ Precis Oncol 4: 7, 2020.

33. Shi Y, Fang N, Li Y, Guo Z, Jiang W, He Y, Ma Z and Chen Y: Circular RNA LPAR3 sponges microRNA-198 to facilitate esophageal cancer migration, invasion, and metastasis. Cancer Sci 111: 2824-2836, 2020
34. Wang X, Chen Y, Liu W, Liu T and Sun D: Hsa_circ 0128846 promotes tumorigenesis of colorectal cancer by sponging hsa-miR-1184 and releasing AJUBA and inactivating Hippo/YAP signalling. J Cell Mol Med 24: 9908-9924, 2020.

35. Choi JH, Kim MJ, Park YK, Im JY, Kwon SM, Kim HC, Woo HG and Wang HJ: Mutations acquired by hepatocellular carcinoma recurrence give rise to an aggressive phenotype. Oncotarget 8: 22903-22916, 2017.

36. Cao Y, Tao Q, Kao X and Zhu X: Hsa-circRNA-103809 promotes hepatocellular carcinoma development via MicroRNA-1270/PLAG1 like zinc finger 2 axis. Dig Dis Sci 66: 1524-1532, 2021.

37. Liu W, Yin C and Liu Y: Circular RNA circ 0091579 promotes hepatocellular carcinoma proliferation, migration, invasion, and glycolysis through miR-490-5p/CASC3 Axis. Cancer Biother Radiopharm: Jul 14, 2020 (Epub ahead of print).

38. Guerrieri F, Belloni L, D'Andrea D, Pediconi N, Le Pera L, Testoni B, Scisciani C, Floriot O, Zoulim F, Tramontano A and Levrero M: Genome-wide identification of direct $\mathrm{HBx}$ genomic targets. BMC Genomics 18: 184, 2017.

39. Levrero M and Zucman-Rossi J: Mechanisms of HBV-induced hepatocellular carcinoma. J Hepatol 64 (Suppl 1): S84-S101, 2016.

(i) $(9)$ This work is licensed under a Creative Commons

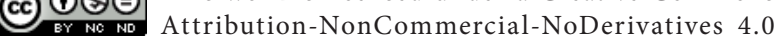
International (CC BY-NC-ND 4.0) License. 